\subsection{2.-19.02.2000}

Dresden

Deutschland

\subsection{2.-19.02.2000 \\ St. Gallen}

Schweiz

\subsubsection{0}

Mainz

Deutschland

\subsection{3.-24.03.2000}

Genf

Schweiz

01.04-05.04.2000

Fiuggi

Italien

12.04.-15.04.2000 Mönchengladbach Deutschland

\subsubsection{0 \\ Halle}

Deutschland

19.05.-21.05.2000

Offenbach

Deutschland

13.06.-17.06.2000

München

Deutschland

\subsection{6.-24.06.2000 \\ Basel}

Schweiz

\subsection{6.-24.06.2000}

Meran

Italien

05.07.-08.07.2000

Lugano

Schweiz

\subsection{9.-08.09.2000}

Washington, D.C.

USA

\subsection{9.-09.09.2000}

Luzern oder Lugano

Schweiz

01.10.-03.10.2000

Landshut

Deutschland
29. Jahrestagung der Deutschen Gesellschaft für

Psychosomatische Gynäkologie und Geburtshilfe

Tumor Prevention and Genetics 2000

1st International Conference and 5th Annual Meeting of

The International Society of Cancer Chemoprevention (ISCaC)

\section{Deutschsprachiges Symposium:}

"Dreidimensionale Sonographie in der Gynäkologie und Geburtshilfe»

3è Cours d'Urogynécologie

2è Journée AUG

16th International Congress 'The Fetus as a Patient'

\section{Operativer Workshop}

Frühjahrstagung der Medizinisch-Wissenschaftlichen Gesellschaft für Gynäkologie und Geburtshilfe in Sachsen-Anhalt

172. Tagung der Mittelrheinischen Gesellschaft für Geburtshilfe und Gynäkologie

53. Kongress der Deutschen Gesellschaft für Gynäkologie und Geburtshilfe (DGGG)

\section{EAGO 2000}

15th European Congress of Gynaecology and Obstetrics (EAGO/EBCOG)

Jahrestagung der Österreichischen Gesellschaft für Gynäkologie und Geburtshilfe (ÖGGG)

\section{Senologie 2000}

Erster gemeinsamer Senologie Kongress der Deutschen, Österreichischen und Schweizer Gesellschaft für Senologie

16th FIGO World Congress of Gynecology and Obstetrics

Jahresversammlung der Schweizerischen Gesellschaft für Gynäkologie und Geburtshilfe (SGGG)

\section{Tagung der Bayerischen Gesellschaft für Geburtshilfe und} Frauenheilkunde e.V.
Auskunft: Gemeinschaftspraxis

Dres. Hellmann \& Schuster,

Rudolf-Renner-Strasse 37, D-01159 Dresden

Tel.: +49-351-421-54-32; Fax: +49-351-458-57-13

Auskunft: Conference Secretariat

'TUP 2000', Mrs. Beatrice Nair,

Center for Tumor Detection and Prevention (ZeTuP),

Rorschacherstr. 150, Postfach, CH-9006 St. Gallen

Tel: +41-71-243-00-32; Fax: +41-71-245-68-05

E-Mail: eso-d-@sg.zetup.ch; web: http://www.zetup.ch

Auskunft: Prof. Dr. E. Merz, (DEGUM-Stufe III),

Leiter der Sektion Gynäkologie und Geburtshilfe,

Universitäts-Frauenklinik Mainz,

Langenbeckstr. 1, D-55101 Mainz

Tel.: +49-6131-177-311; Fax: +49-6131-173-415

Auskunft: PD Dr. med. A. Weil,

Dept. de Gynécologie et Obstétrique,

HUG, Genève, Suisse

Tel.: +41-22-382-74-10, Fax: +41-22-382-44-24

Auskunft: Organizing Secretariat,

2nd Institute of Obstetrics and Gynecology,

University 'La Sapienza',

Viale Regina Elena 324, I-00161 Rome (Italy)

Tel. +39-6-446-04-84; Fax: +39-6-446-91-28

E-Mail: perinat@flashnet.it

Auskunft: Prof. Dr. med. B.R. Muck,

Chefarzt der Frauenklinik, Ev. Krankenhaus Bethesda, Ludwig-Weber-Str. 15, D-41061 Mönchengladbach Tel.: +49-2161-981-23-30

Auskunft: Sekretariat der Klinik für Gynäkologie der Martin-Luther-Universität Halle-Wittenberg, H. Friedrich, Magdeburger Str. 24, D-06097 Halle Tel.: +49-345-557-4127; Fax: +49-345-557-4017

Auskunft: Congress Organisation

C. Schäfer, Karl-Theodor-Str. 64, D-80803 München Tel.: +49-89-307-10-11; Fax: +49-89-307-10-21; E-Mail: sandra.heussner@cocs.de

Auskunft: Congress Project Management gmbH Letzter Hasenpfad 61, D-60598 Frankfurt Tel: +49-69-6090-9531; Fax: +49-69-6090-9540 E-Mail: cpm.sachs.ffm@t-online.de web: http://www.dggg-kongress-2000.de

Auskunft: EAGO 2000, c/o AKM Congress Service, Clarastr. 57, P.O. Box, CH-4005 Basel

Tel: +41-61-686-77-11; Fax: +41-61-686-77-88; E-Mail: info@akm.ch

Auskunft: Congress Management, Wallachgasse 8, A-1230 Wien

Tel.: +43-1-698-1000-60; Fax: +43-1-698-1000-66

Auskunft: NICO ENTERPRISE SA,

CP 59, CH-6929 Gravesano/Lugano

Tel.: +41-91-605-1723; Fax: +41-91-605-4675; E-Mail:info@senologie2000.org

Auskunft: FIGO Secretariat, 27 Sussex Place, Regent's Park, London NW4 1RG(UK) Tel.: +44-171-723-2951; Fax: +44-171-258-0737; E-Mail: secret@figo.win-uk.net

\section{Auskunft: PD Dr. R.A. Steiner,}

Chefarzt, Kant. Frauenspital Fontana,

Lürlibadstrasse 118, CH-7000 Chur

Tel.: +41-81-254-8128; Fax: +41-81-254-8130;

E-Mail: Rolf.Steiner@san.gr.ch

\section{Auskunft: Prof. Dr. Hubert Elser,}

Chefarzt der gynäkologisch-geburtshilflichen

Abteilung, Achdorferweg 3, D-84046 Landshut

Tel.: +49-871-404-27-07; Fax: +49-871-404-16-52

\section{KARGER}

Fax + 41613061234 E-Mail karger@karger.ch www. karger.com

\section{(c) 1999 S. Karger AG, Basel}

Accessible online at:

www.karger.com/journals/ggr 